\title{
Candidiasis esofágica en pacientes inmunocompetentes: Estudio clínico e inmunológico
}

\author{
Claudia Cortés $\mathbf{M}^{\mathbf{1}}$, Danny Oksenberg $\mathbf{R}^{\mathbf{2}}$, \\ Alejandro Afani $S^{3}$, Carlos Defilippi $C^{2}$, Ana María Madrid $S^{2}$. \\ Clinical and immunological study of \\ 10 immunocompetent patients with \\ esophageal candidiasis
}

Background: Esophageal candidiasis is associated with conditions that cause an immune depression. It is a defining disease for AIDS, is observed in poorly controlled diabetics, in patients with renal or hepatic failure, in patients with cancer and in subjects using medications causing immunosuppression or broad spectrum antimicrobials. Aim: To report the features of 10 immunocompetent patients with esophageal candidiasis. Patients and methods: Six males and four females aged between 48 and 82 years, without conditions associated with immunosuppression, in whom an esophageal candidiasis was found on an upper gastrointestinal endoscopy. Delayed skin hypersensitivity to eight antigens, Iymphocyte subpopulations, yeast phagocytosis and neutrophil chemotaxis were measured. Results: Six patients had a low CD4 Iymphocyte count and seven had a low CD8 count. Seven patients were anergic on skin hypersensitivity challenge. Yeast phagocytosis was abnormal in one patient and neutrophil chemotaxis was abnormal in two. Humoral immunity was normal in all subjects. All patients were treated with oral fluconazole in doses of $150 \mathrm{mg} /$ day for 14 days, with complete resolution of candidiasis in all. Conclusions: Patients with esophageal candidiasis, have frequent alterations of cellular immunity, that must be diagnosed and treated (Rev Méd Chile 2004; 132: 1389-94).

(Key Words: Candidiasis, esophageal; Immunity, cellular; Immunocompetence)

Recibido el 24 de marzo, 2004. Aceptado en versión corregida el 2 de septiembre, 2004.

${ }^{1}$ Sección Medicina Interna, 2Centro de Gastroenterología y 3Sección Inmunología, Hospital Clínico Universidad de Chile

$\mathrm{C}$ andida albicans es una levadura de alta prevalencia en la población humana. Es la principal levadura oportunista patógena y la causa más importante de esofagitis infecciosa. Se le considera ubicua en el ambiente hospitalario, ya sea en el aire, superficies inertes como pisos o techos y también en los alimentos ${ }^{1,2}$.

Correspondencia a: Dr. Danny Oksenberg R. Santos Dumontt 999. Centro de Gastroenterología. Fax: 56-2 2691340. E mail: dannyoksenberg@hotmail.com
La colonización por Candida spp es variable según el lugar anatómico en el cual se ha estudiado, presentando una alta prevalencia en los extremos del tubo digestivo. Así, se puede aislar frecuentemente de la cavidad oral, donde es posible detectarla en 30 a $40 \%$ de individuos sanos ${ }^{3}$.

El principal factor predisponente a la colonización y posterior infección por Candida spp es el estado inmunológico de base. Este puede alterarse 
en variadas situaciones; entre ellas, es posible destacar: 1. Las dependientes del huésped, como son: las edades extremas, la alteración de la función fagocítica (ya sea cualitativa o cuantitativa), la presencia de diversas patologías (diabetes mellitus, insuficiencia renal o hepática, neoplasias y algunas enfermedades endocrinológicas), también infección por virus de inmunodeficiencia humana (VIH) y condiciones que alteran la integridad de las mucosas: quimio o radioterapia, trauma o cirugía de gran envergadura, 2. Factores exógenos como uso crónico de esteroides orales 0 inhalatorios, el uso prolongado de antimicrobianos de amplio espectro y la administración de inmunosupresores ${ }^{1,3-5}$.

El objetivo de nuestro trabajo fue estudiar una serie de casos con infección esofágica por Candida spp, en ausencia de los factores de riesgo antes mencionados, los que fueron exhaustivamente analizados desde el punto de vista clínico e inmunológico, en busca de una probable etiología.

\section{PACIENTES Y MÉTODOS}

Se incluyeron 10 pacientes de manera prospectiva, en el período comprendido entre los años 1999 y 2002, todos los cuales presentaron candidiasis esofágica (CE), sospechada por endoscopia digestiva alta y demostrada por observación microscópica directa al fresco.

El estudio incluyó a seis hombres y cuatro mujeres, entre 48 y 82 años, con un promedio de edad de 60,3 años (desviación estándar: \pm 10,6 años).

Todos los pacientes consultaron a nuestro centro de gastroenterología y fueron evaluados mediante endoscopia digestiva alta. Todos presentaron uno o más de los siguientes síntomas: náuseas, vómitos, disfagia, anorexia, pirosis, epigastralgia o baja de peso.

Ningún paciente presentaba evidencia de candidiasis orofaríngea al momento de la endoscopia, ni tenía antecedentes de infecciones por cándida en ocasiones anteriores.

Los criterios de exclusión fueron: diabetes mellitus, insuficiencia renal o hepática, neoplasia, HIV (+) y uso previo de inmunosupresores o esteroides en cualquiera de sus formas, así como de antimicrobianos de amplio espectro en los 15 días precedentes.
Se investigó la presencia de VIH en dos oportunidades, separadas por 3 meses $\left(\operatorname{VIDAS}^{\circledR}\right.$ HIV DUO Biomerieux, 4⿳亠丷厂 generación). Se realizó hemograma completo, pruebas hepáticas, perfil bioquímico, creatinina, electrolitos plasmáticos, examen de orina completa, anticuerpos antinucleares en Hep II (ANA), factor reumatoide (FR), radiografía de tórax y ecografía abdominal.

Se cuantificaron los niveles de inmunoglobulinas (Ig), A, G y M.

Para el análisis de la inmunidad celular se estudiaron las subpoblaciones linfocitarias CD4 y CD8 mediante citometría de flujo (Cytometer FACSCalibur E3717), multitest (estudio de hipersensibilidad retardada), pruebas de fagocitosis en levadura y pruebas para quimiotaxis de neutrófilos.

\section{Resultados}

Los principales resultados del estudio inmunológico se observan en la Tabla 1.

Las enfermedades más frecuentemente asociadas fueron: depresión en cuatro casos, gota e hipertensión arterial en dos, vitiligo y psoriasis en un paciente.

Sólo dos pacientes se encontraban utilizando inhibidores de la bomba de protones (IBP), a dosis habituales, durante el estudio. Los demás pacientes no habían recibido con anterioridad al diagnóstico.

Todos los pacientes, salvo uno, presentaron un recuento leucocitario total dentro de rangos normales: promedio $(\overline{\mathrm{x}}) 5.920 / \mathrm{mm}^{3}$ con un rango $(\mathrm{r})$ $3.400-8.100 / \mathrm{mm}^{3}$; un paciente tenía una discreta leucopenia $\left(3.400 \mathrm{cels} / \mathrm{mm}^{3}\right)$. El recuento linfocitario promedio fue de $1.241 / \mathrm{mm}^{3}$ con un $\mathrm{r}=690$ $2.016 / \mathrm{mm}^{3}$. La subpoblación de linfocitos T CD4 se encontró disminuida en 6 pacientes $(\bar{x}=497 /$ $\mathrm{mm}^{3}, \mathrm{r}=220-807$ ) (Figura 1), y la de linfocitos $\mathrm{T}$ CD8, en 7 pacientes $\left(\bar{x}=362 / \mathrm{mm}^{3}, r=154-780\right)$ (Figura 2).

El estudio de hipersensibilidad retardada, realizado mediante Multitest, resultó anérgico en siete pacientes y normal en los otros tres. La fagocitosis de levaduras se encontraba normal en nueve pacientes y disminuida en uno (paciente \# 7). El test de quimiotaxis de neutrófilos fue normal en ocho pacientes y discretamente alterado en dos de ellos (pacientes \# 1 y 2). 
Tabla 1. Resultados de estudio inmunológico

\begin{tabular}{|lcccccccccccc|}
\hline Paciente & 1 & 2 & 3 & 4 & 5 & 6 & 7 & 8 & 9 & 10 & Prom & DE \\
\hline Edad (años) & 59 & 55 & 63 & 48 & 82 & 55 & 55 & 62 & 50 & 74 & 60,4 & 10,60 \\
Sexo & $\mathrm{M}$ & $\mathrm{F}$ & $\mathrm{M}$ & $\mathrm{F}$ & $\mathrm{F}$ & $\mathrm{M}$ & $\mathrm{F}$ & $\mathrm{F}$ & $\mathrm{M}$ & $\mathrm{F}$ & & \\
Leucocitos & 5.300 & 7.150 & 6.950 & 6.900 & 8.100 & 4.900 & 6.400 & 3.400 & 4.500 & 5.600 & 5.920 & $1.430,3$ \\
Linfocitos & 1.007 & 1.638 & 1.807 & 690 & 992 & 931 & 768 & 1.698 & 871 & 2.016 & $1.241,8$ & 490,4 \\
CD4 & 412 & 807 & 710 & 220 & 450 & 372 & 269 & 760 & 260 & 724 & 498,4 & 229,0 \\
CD8 & 223 & 219 & 700 & 270 & 308 & 223 & 154 & 540 & 321 & 780 & 373,8 & 219,8 \\
VHS & 15 & 20 & 42 & 15 & 48 & 18 & 21 & 13 & 31 & 11 & 23,4 & 12,7 \\
Fag lev & $83 \%$ & $100 \%$ & $76 \%$ & $90 \%$ & $95 \%$ & $100 \%$ & $36 \%$ & $100 \%$ & $100 \%$ & $95 \%$ & $88 \%$ & - \\
MDSA & 2,7 & 1,8 & 1,3 & 1,3 & 1,35 & 0,9 & 1,3 & 1,45 & 1,75 & 1,33 & 1,518 & 0,49 \\
MDSN & 2,7 & 2,1 & 1,1 & 1,2 & 1,32 & 0,9 & 1,25 & 1,4 & 1,65 & 1,3 & 1,492 & 0,54 \\
MA & 1,45 & 0,85 & 0,6 & 0,7 & 0,73 & 0,55 & 0,8 & 0,63 & 0,8 & 0,78 & 0,789 & 0,25 \\
IQSA & 1,86 & 2,09 & 1,52 & 1,86 & 1,88 & 1,63 & 2,08 & 1,09 & 2,18 & 2,02 & 1,82 & 0,33 \\
IQSN & 1,86 & 2,66 & 1,55 & 1,71 & 1,75 & 1,63 & 2,11 & 1,12 & 2,06 & 2,03 & 1,84 & 0,42 \\
Multi test & normal & anérgico & anérgico & normal & anérgico & anérgico & anérgico & anérgico anérgico & normal & - & - \\
Ig A & 205 & 173 & 377 & 242 & 242 & 165 & 500 & 341 & 276 & 320 & 284,1 & 103,37 \\
Ig G & 1.050 & 980 & 1.100 & 1.360 & 1.480 & 1.180 & 1.473 & 1.410 & 730 & 1.050 & $1.181,3$ & 245,82 \\
Ig M & 98 & 62 & 180 & 96 & 51 & 296 & 121 & 107 & 231 & 102 & 134,4 & 77,82 \\
\hline
\end{tabular}

Abreviaciones y rangos normales:

IQSA: Indice quimiotáctico en suero autólogo: 1,08-2,5 mm Ig A: 70-312 U/ml IQ SN: Indice quimiotáctico en suero normal: $1,08-2,5 \mathrm{~mm}$ Ig G: $639-1.350 \mathrm{U} / \mathrm{ml}$ MDSA: Migración dirigida a suero autólogo: 0,52-1,75 mm Ig M: 56-352 U/ml MDSN: Migración dirigida a suero normal: 0,52-1,75 mm Fag lev: Fagocitosis de levaduras: 85-100\% MA: Migración al azar: 0,48-0,70 mm

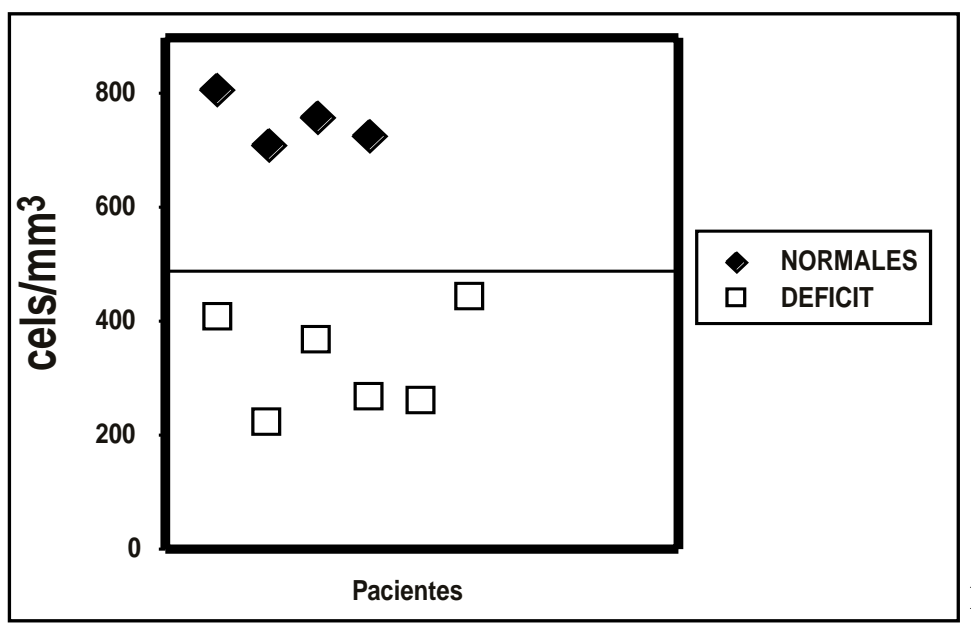

FiguRA 1. Recuento de linfocitos T CD4. 


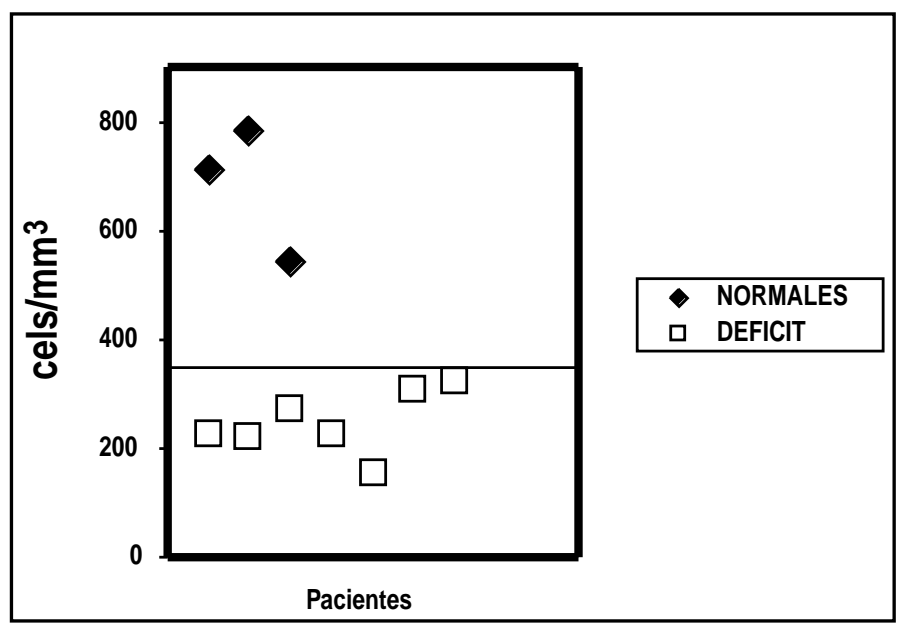

FiguRA 2. Recuento linfocitos T CD8.

Los valores de Ig A, G y M, fueron normales, con excepción de dos pacientes, que presentaron un aumento menor a dos veces de la Ig A, que en forma aislada nos parece clínicamente no relevante, como así mismo, un paciente presentó un valor de Ig $\mathrm{G}$ el doble del valor normal, cuya interpretación es incierta.

Los exámenes generales, radiografía de tórax y ecografía abdominal fueron normales en todos los pacientes. Los ANA y el FR fueron negativos en todos los casos.

Todos los pacientes fueron tratados con Fluconazol oral, $150 \mathrm{mg}$ diarios por 14 días, con mejoría en la totalidad de ellos, siendo demostrada mediante control endoscópico. Luego del tratamiento y con el estudio inmunológico completo, los pacientes fueron derivados a inmunología, en donde los 7 pacientes con disminución de los recuentos linfocitarios, recibieron Methisoprinol (Isoprinosine ${ }^{\circledR}$ ) $500 \mathrm{mg}$ cada 8 horas vía oral, en días alternos, por tres meses y un inmunoestimulante (en base a fracción ribosomal de Klebsiella pneumoniae, Diplococcus pneumoniae, Streptococcus pyogenes y Haemophilus influenzae, más fracción membranal de Klebsiella pneumoniae [Ribomunyl $\left.^{(\mathrm{r})}\right]$ ) en las dosis recomendadas ${ }^{6}$.

Se realizó seguimiento endoscópico al mes y al año del tratamiento, sin recidiva en ninguno de los casos. Hasta la fecha, y con seguimiento de entre 2 y 4 años de todos los pacientes, no se ha comprobado recidiva clínica de la CE. En un paciente se diagnosticó una gamapatía monoclo- nal de significado incierto, sin progresión a mieloma múltiple, y en otro se diagnosticó un carcinoma espinocelular de la piel, patología no demostrada como predisponente a CE. Los linfocitos T CD4 y CD8 se normalizaron en todos los casos tratados y no fue factible el seguimiento con multitest, por discontinuación de la técnica en nuestro hospital.

\section{DISCUSIÓN}

Nuestro estudio se refiere a la presencia de CE en una serie de casos, en ausencia de una inmunodeficiencia clínicamente evidente. Sin embargo, en la casi totalidad de los pacientes estudiados, se logra demostrar la existencia de alteraciones de la inmunidad celular, mediante el estudio dirigido.

La mayor parte de las publicaciones relacionadas con $\mathrm{CE}$, se refieren a pacientes inmunocomprometidos, especialmente pacientes portadores de VIH. Las publicaciones que excluyen sujetos VIH positivo son escasas y en general logran identificar algún tipo de patología o factor predisponente de inmunosupresión. En nuestro medio, en el único estudio que aborda el tema, Maggiolo y Klinger ${ }^{7}$ presentaron 25 casos de CE, de los que sólo 3 fueron catalogados como sanos; en los 22 restantes se encontró alguna patología predisponente, principalmente neoplásica, uso de tratamiento esteroidal o inmunosupresor. No obstante, en otras series, se describen pacientes a los que 
no fue posible encontrar ningún factor predisponente ${ }^{8-10}$. En este trabajo se consideró criterio de exclusión patologías reconocidamente inmunosupresoras y, a partir de ello, se efectuó un completo estudio inmunológico humoral y celular.

La presencia de síntomas en todos los pacientes de nuestra serie concuerda con la mayoría de los trabajos en inmunocompetentes, relativos al hecho que la CE es casi invariablemente sintomática. Sin embargo, en las revisiones de pacientes mayoritariamente inmunosuprimidos, sólo un tercio de ellos presentaron síntomas ${ }^{3,11}$. Esta diferencia es atribuible a la poca capacidad para montar una adecuada respuesta inmune, en los pacientes inmunocomprometidos.

Ninguno de los pacientes era portador de inmunodeficiencia evidente; si bien nuestro algoritmo diagnóstico no abarcó una investigación exhaustiva de patologías oncológicas, el seguimiento a 4 años plazo descarta razonablemente esta causa de inmunosupresión, en 8 de los 10 pacientes. En uno de los casos se confirmó una gamapatía monoclonal de significado incierto, que hasta la fecha no ha progresado a mieloma múltiple. Otro paciente presentó un carcinoma espinocelular de piel 14 meses después del diagnóstico de $\mathrm{CE}$, patología no demostrada como predisponente a CE.

En cuanto a la asociación entre el uso de inhibidores de la bomba de protones (IBP) y la presencia de infección esofágica por Candida spp, en nuestra serie de casos sólo dos pacientes refirieron utilizarlos por al menos 2 meses, previo al inicio de los síntomas esofágicos, los pacientes restantes niegan el uso de IBP con anterioridad. En la literatura la información es escasa y poco concluyente. Hasta el momento no existen estudios de casos y controles en pacientes que carezcan de antecedentes de inmunodepresión. Pese a ello, es razonable pensar que la alteración del $\mathrm{pH}$ gástrico causada por estas drogas, pueda alterar el balance de la microflora en este segmento del tubo digestivo, llevando a una más fácil colonización y posterior infección por cándida. También se debe considerar que el pH ácido del reflujo normal facilita la eliminación de gérmenes, al verse elevado este $\mathrm{pH}$ por el uso de IBP, este mecanismo se ve comprometido. No obstante, considerando el uso masivo en el mundo de los IBP y la muy baja prevalencia de CE en inmuno- competentes, es plausible pensar que no hay asociación real entre ambas circunstancias 3,12,13.

En seis de los diez pacientes estudiados, se pesquisó un déficit en el recuento de linfocitos CD4, ( $\overline{\mathrm{x}}$ de estos casos: $306 / \mathrm{mm}^{3}$ ), comparado con un valor $\overline{\mathrm{x}}$ de $666 / \mathrm{mm}^{3}$ del grupo sin déficit y con un de $\bar{x} 497$ células $/ \mathrm{mm}^{3}$ del grupo total. También fue posible pesquisar una disminución del recuento total de linfocitos T CD8 en 7 casos, seis de los cuales correspondieron a los pacientes con linfocitos T CD4 disminuidos. Los valores encontrados correspondieron a un $\bar{x}=245 / \mathrm{mm}^{3}$, para el grupo con déficit ( 7 casos), comparado con un $\bar{x}=676 / \mathrm{mm}^{3}$ del grupo normal (3 casos) y un $\overline{\mathrm{x}} 362 / \mathrm{mm}^{3}$ del grupo total.

Destaca también que siete pacientes presentaron anergia en el multitest, evidenciando una alteración cualitativa de la inmunidad celular.

Estas alteraciones cuantitativas o cualitativas de la inmunidad celular, fueron la principal evidencia de inmunodeficiencia aislada en estos pacientes, logrando ser demostrada en 9 de los diez pacientes. Esta alteración en la inmunidad celular es de carácter inespecífico, pero pensamos, revela una condición predisponente a la $\mathrm{CE}$, que debe ser diagnosticada, tratada y segui$\mathrm{da}$, para prevenir la recurrencia y eventualmente la aparición futura de una inmunodeficiencia específica. Es plausible también, que la presencia de enfermedad de la esfera psicológica, asociada en 4 de nuestros enfermos, todos los cuales tuvieron un déficit inmunitario, favorezca esta condición, por mecanismos no bien aclara$\operatorname{dos}^{14}$.

En el paciente en el cual no fue posible objetivar ninguna deficiencia de su mecanismo inmunológico celular, es posible que la alteración se encuentre a nivel de la inmunidad de las mucosas, o bien, que las alteraciones de la inmunidad celular presenten un carácter fluctuante 0 intermitente.

De esta forma, y basados en los resultados del presente estudio, aportamos una nueva propuesta, consistente en la necesidad de un estudio inmunológico celular, fundamentalmente subpoblaciones linfocitarias y multitest, al enfrentarse a una $\mathrm{CE}$ en un paciente inmunocompetente, por cuanto las alteraciones de este sistema parecieran ser frecuentes, benignas y susceptibles de ser tratadas y revertidas. 


\section{REFERENCIAS}

1. VÁzquez J, Sobel J. Mucosal candidiasis. Infect Dis Clin North Am 2002; 16: 793-820.

2. Andersen LI, Frederiksen HJ, Appleyard M. Prevalence of esophageal Candida colonization in a Danish population: special reference to esophageal symptoms, benign esophageal disorders and pulmonary disease. J Infect Dis 1992; 165: 389-92.

3. Chocarro Martínez A, Galindo Tobal F, RuizIrastroza G, González López A, Álvarez Navia F et AL. Risk factors for esophageal candidiasis. Eur J Clin Microbiol Infect Dis 2000; 19: 96-100.

4. TRIER JS, BJORKMAN DJ. Esophageal, gastric and intestinal candidiasis. Am J Med 1984; 77: 39-43.

5. Simon M, Houser W, Smith K, Long M. Esophageal candidiasis as a complication of inhaled corticosteroids. Ann Allergy Asthma Immunol 1997; 79: 333-8.

6. Сцот J. Pharmacology of ribosomal immunotherapy. Drugs 1997; Suppl 1: 33-6.

7. Maggiolo P, Kunger J. Candidiasis esofágica, experiencia en 25 casos. Rev Méd Chile 1981; 109: 1163-7.
8. Kodsi B, WickRemesinghe P, Kozinn P, Iswara $\mathrm{K}$, GoLDBERG P. Candida esophagitis, a prospective study of 27 cases. Gastroenterology 1976; 71: 715-9.

9. Ortuño JA, Tovar A, Ruiz J, García A. Candidiasis esofágica en pacientes VIH negativos. Revista Española de Enfermedades Digestivas 1997; 89: 503-10.

10. Kimura H, Karachi M, Tsukioka Y, Minami M, Itou M, FujII H ET AL. Esophageal stricture secondary to candidiasis without underlying diseases. J Gastroenterol 1995; 30: 508-11.

11. Samonis G, Skordils P, Maraki S, Datseris G, Toloudis P, Chatzinikolaou I et al. Oropharyngeal candidiasis as a marker for esophageal candidiasis in patients with cancer. Clin Infect Dis 1998; 27: 283-6.

12. LARNER AJ, LeNDRUM R. Esophageal candidiasis after omeprazol therapy. Gut 1992; 33: 860-1.

13. Sood A, Sharma M, Jain NP, Chawla LS, Kumar R. Esophageal candidiasis following omeprazole therapy: a report of two cases. In: J Gastroenterology 1995; 14: 71-2.

14. DutTa SK, AL-IBrahim MS. Immunological studies in acute pseudomembranous esophageal candidiasis. Gastroenterology 1978; 75: 292-6. 